\title{
Controversies in total knee arthroplasty: Cochrane evidence or surgeons' philosophy?
}

\author{
Patrick Sadoghi ${ }^{1}$
}

Received: 4 June 2015 / Accepted: 26 June 2015 / Published online: 2 July 2015

(C) European Society of Sports Traumatology, Knee Surgery, Arthroscopy (ESSKA) 2015

Total knee arthroplasty (TKA) is a frequently done and highly successful treatment for end-stage osteoarthritis of the knee joint [1]. The surgeon has to choose from different options such as the adequate cementation technique, a fixed or mobile bearing, retention or sacrifice of the posterior cruciate ligament, or the use of continuous passive motion (CPM). Although research has focused on the improvement of TKA within decades, there are still substantial aspects unclear and not fully understood [1]. From a technical point of view, these aspects seem to offer very different treatment methods and their different clinical impact should be obvious. However, this is not the case.

The level of evidence pyramid declares that highest levels of evidence are gained from meta-analysis of level I trials. Even beyond that, the Cochrane review database is a useful tool to find out the true meaning of a procedure. It should be the ideal tool to answer unclear questions on this very common procedure. Interestingly, even Cochrane review articles cannot give definite recommendations for important treatment options, and therefore, these are more choices of our philosophy as knee surgeons:

1. There is an option of cemented versus cementless fixation, where Cochrane finds a smaller displacement of the cemented tibial component but also a greater risk of future aseptic loosening in comparison with cementless fixation without a recommendation [2].

2. Another option is the use of fixed versus mobile bearings in TKA, where Cochrane reports that mobile and

Patrick Sadoghi

patricksadoghi@gmx.at

1 Department of Orthopedic Surgery, Medical University of Graz, Auenbruggerplatz 5, 8036 Graz, Austria fixed bearing after cruciate retaining TKA have similar effects in pain, clinical outcome, functional scores, quality of life, revision surgery, mortality, and reoperation rate without a preference [3].

3. We surgeons might choose retention versus sacrifice of the posterior cruciate ligament in TKA, where Cochrane finds no differences with respect to range of motion, pain, or clinical and radiological outcomes and states that differences are not clinically relevant [4].

4. And we might use continuous passive motion (CPM) after TKA, where Cochrane states that CPM has no clinically important effects on active ROM, pain, function, or quality of life to justify its routine use [5].

Therefore, despite obvious technical differences and highest levels of evidence including Cochrane reviews, we surgeons are the ones to choose from these options based on our philosophy and cannot comment using for or against specific methods using hard criteria. We face a certain status-when even Cochrane Evidence leads to Philosophy.

\section{References}

1. Carr AJ, Robertsson O, Graves S, Price AJ, Arden NK, Judge A, Beard DJ (2012) Knee replacement. Lancet 379(9823):1331-1340

2. Nakama GY, Peccin MS, Almeida GJ, Lira Neto Ode A, Queiroz AA, Navarro RD (2012) Cemented, cementless or hybrid fixation options in total knee arthroplasty for osteoarthritis and other non-traumatic diseases. Cochrane Database Syst Rev 10:CD006193

3. Hofstede SN, Nouta KA, Jacobs W, van Hooff ML, Wymenga AB, Pijls BG, Nelissen RG, Marang van de Mheen PJ (2015) Mobile bearing vs fixed bearing prostheses for posterior cruciate retaining total knee arthroplasty for postoperative functional 
status in patients with osteoarthritis and rheumatoid arthritis. Cochrane Database Syst Rev 2:CD003130

4. Verra WC, van den Boom LG, Jacobs W, Clement DJ, Wymenga AA, Nelissen RG (2013) Retention versus sacrifice of the posterior cruciate ligament in total knee arthroplasty for treating osteoarthritis. Cochrane Database Syst Rev 10:CD004803
5. Harvey LA, Brosseau L, Herbert RD (2014) Continuous passive motion following total knee arthroplasty in people with arthritis. Cochrane Database Syst Rev 2:CD004260 